\title{
A Modified Multicarrier Modulation Binary Data Embedding in Audio File
}

\author{
Gelar Budiman ${ }^{1}$, Andriyan B. Suksmono ${ }^{2}$, Donny Danudirdjo ${ }^{3}$, \\ Koredianto Usman ${ }^{4}$, and Dong Hwan Shin $^{5}$ \\ Electrical Engineering Faculty, Telkom University ${ }^{\mathbf{1 , 4}}$ \\ Graduate School of Electrical and Informatics Engineering Bandung Technology Institute ${ }^{\mathbf{2 , 3}}$ \\ Markany Inc. ${ }^{5}$, \\ gelarbudiman@telkomuniversity.ac.id ${ }^{1}$, suksmono@stei.itb.ac.id ${ }^{2}$, donny@stei.itb.ac.id ${ }^{3}$, \\ koredianto.usman@gmail.com ${ }^{4}$,dhshin@markany.com ${ }^{5}$
}

Abstract: Information hiding of data in an audio file is an important thing that the media can be recognized by its ownership. The hidden information would be the important information which describes the copyright of the audio file. In this research, the binary data is inserted or hidden into the audio file by multicarrier modulation technique data hiding. The encoded binary data is modulated by multicarrier frequencies before embedding into the host audio. Data hiding capacity with this technique can achieve up to 40 bits per second in file mode with good audio imperceptibility. It also achieves perfect robustness for type of attack noise addition, linear speed change, multi band equalization, and echo addition. And this technique is still acceptable for type of attack, such as resampling attack, MP3 compression attack and filtering attack for cut off frequency more than $10 \mathrm{kHz}$ regarding to the BER less than $10 \%$.

Keywords: Modified multicarrier modulation, copyright, binary data, extraction, insertion, imperceptibility, attack

\section{Introduction}

Multicarrier modulation is a technique to modulate a baseband signal into several subcarrier at different frequencies in the same time. Prasad [1] said that OFDM basic principle is to split datastream into lower datastream transmitting simultaneously over several subcarriers. This principle is also called multicarrier modulation. The research about multicarrier modulation technique has been previously published on many papers and books. Nicola Marchetti [2] said that OFDM promises high data rate capability and uses available spectrum efficiently, which is a main reason OFDM was accepted by present telecommunication generation system.

Data hiding by the OFDM method was already presented by Somnath [3]. His research is about hiding the grayscale image in OFDM signal where the image is read pixel by pixel and then converted the binary data into a complex number format and inserted the number via Quantized Index Modulation. In [4] Amirtharajan presented the paper describing the multicarrier steganography. He presented the image hiding by Spread Spectrum method combined with the Modulated Multicarrier signal in orthogonal radio frequency, but the robustness and imperceptibility were not explained. Shishkin [5] presented audio watermarking for Electronic Radiotelephone Identification in OFDM-based. He used Quantization Index Modulation as embedding method in frequency domain, but he didn't describe the imperceptibility and robustness. Garcia-Hernandez [6] proposed a high payload data-hiding scheme for audio signals in OFDM-based. The method which he used was to change the phase component of audio signal via a reduced-arc of M-order Phase Shift Keying (MPSK). It was modulated on selected frequency of the audio signals, however he only described the imperceptibility and capacity. The robustness was not described clearly.

In this paper, we propose audio watermarking by multicarrier modulation method which the subcarrier signal are not orthogonal. This multicarrier watermarking is a watermarking process in which the hidden data is modulated by multicarrier by many frequencies from 0 to $22 \mathrm{kHz}$. The process of watermarking is not only multicarrier modulation of the hidden data, but also

Received: January $18^{\text {th }}, 2016$. Accepted: December $19^{\text {th }}, 2016$ 
there are several subprocessing before modulation processing and embedding the data. The imperceptibility, capacity, and robustness is described in this proposed method. The topic of this paper is modified and improved version of our previous paper in [7].

This paper is organized as follow: section 2 describes the watermarking model of multicarrier audio watermarking, section 3 presents multicarrier modulation embedding process, section 4 describes the extraction performance which analyzed the data extraction quality after being attacked and the capacity of embedded data, while the conclusion is presented in section 5 .

\section{Watermarking Model}

The total watermark payloads of an audio watermarking system (AWS) consists of $\mathrm{N}$ bits hidden data as shown in figure 1. $\mathrm{s}(\mathrm{i})$ is binary watermark which has $\mathrm{N}$ bits content, where $\mathrm{i}$ is binary-based discrete time unit, since $\mathrm{s}(\mathrm{i})$ is in binary form. It is also modulated by multicarrier frequencies before embedding into the host audio. The watermark duration is 1 second with variation length of bits ( $\mathrm{N}$ bits) to be compared and analyzed.

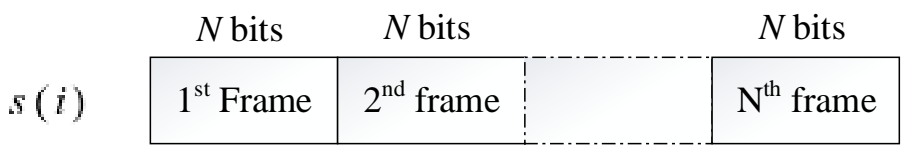

Figure 1. Watermark Payload

In the AWS, a frame is a basic unit of embedding (or extracting) a watermark bit. As shown in figure 1, after the NRZ conversion, watermark bit is modulated by multicarrier modulation and filtered by psychoacoustic filter which will be described at section 3 . The filtered signal is then copied to two branch. One signal is controlled by $A_{1}$ gain. The other signal is filtered by high pass filter before controlled by $\mathrm{A}_{2}$ gain. Finally, the watermarked audio is produced by adding those two signals after controlled by $\mathrm{A}_{1}$ and $\mathrm{A}_{2}$ gains with host audio.

The Audio Watermarking System (AWS) algorithm in this paper is described as following :

1. Generating the information data

2. Convert the data from binary form to NRZ form

3. Processing the binary NRZ data by multicarrier modulation

4. The modulated signal which consist of data is filtered by psychoacoustic filter to decrease the signal level to the non human auditory level.

5. The signal is multiplied by gain $\mathrm{A}_{1}$ as primary multiplier as a controller to hidden data before embedded into the host audio.

6. The signal is also filtered by HPF and multiplied by secondary multiplier $\mathrm{A}_{2}$ and add into the signal from step 5 .

7. Embedding the signal from two branchs into the host audio by additional processing.

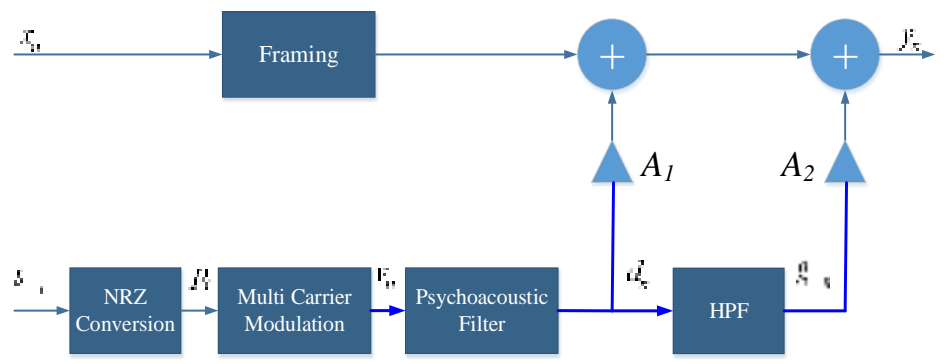

Figure 2. Processing Stages Inside Embedding Process of AWS 
A communication system with multicarrier modulation transmit $\mathrm{N}_{\mathrm{c}}$ binary values source, in parallel on $\mathrm{N}_{\mathrm{r}}$ subcarriers with $\mathrm{N}_{\mathrm{c}}$ frequencies of each subcarrier, $\mathrm{f}_{\mathrm{ij}}$ and are converted to multicarrier symbol duration $\mathrm{T}_{\mathrm{s}}$. Our proposed method of audio watermarking embedding is displayed in figure 2 .

The result of embedding process is given by :

$$
y(n)=x(n)+A_{1} d(n)+A_{2} g(n)
$$

Where

$\mathrm{x}(\mathrm{n})=$ host audio in frame-based

$\mathrm{A}_{1}=$ gain of $\mathrm{d}(\mathrm{n})$

$\mathrm{A}_{2}=$ gain of $\mathrm{g}(\mathrm{n})$

$\mathrm{y}(\mathrm{n})=$ watermarked audio

$\mathrm{d}(\mathrm{n})=$ the multicarrier modulated watermark data

$\mathrm{d}(\mathrm{n})$ is also the result of psychoacoustic filter process as follow :

$d(n)=v(n) * h_{P}(n)$

$\mathrm{v}(\mathrm{n})=$ the multicarrier modulated signal

$\mathrm{h}_{\mathrm{P}}(\mathrm{n})=$ psychoacoustic filter coefficient which will be described in section 3

$\mathrm{g}(\mathrm{n})=$ the highpass signal filtered signal

$\mathrm{g}(\mathrm{n})$ is the result of HPF as follow :

$$
g(n)=d(n) * h_{H P F}(n)
$$

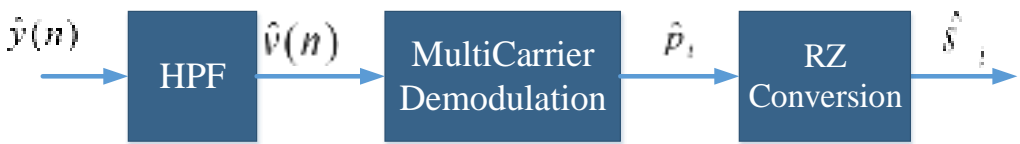

Figure 3. Processing Stages Inside AWS Extraction

The proposed extraction process is displayed in figure 3. A frame is selected from a watermarked audio signal and then it is filtered by HPF before decoded by multicarrier demodulation. High pass filtering is used for removing low frequency signal in which most of watermark information is damaged. After demodulation, the signal is converted back to binary information by RZ conversion.

\section{Multicarrier Modulation Process}

Multicarrier modulation process consists of several subsystems such as : serial to parallel, copier, pulse shaper, oscillator and multiplier, and adder. The sequence of information bits is demultiplexed into several rakes. In each rake every bit is modulated by more than 1 frequency. As displayed in figure 5, the output of each rake after multicarrier modulation, as example output for $\mathrm{i}^{\text {th }}$ rake $\left(\mathrm{q}_{\mathrm{i}}(\mathrm{n})\right)$ is [8]:

$$
q_{i}(n)=p_{i} \sum_{j=1}^{N_{c}} \cos \left(2 \pi f_{i j} n\right)
$$

Thus :

$$
r(n)=\sum_{i=1}^{N r} p_{i} \sum_{j=1}^{N_{c}} \cos \left(2 \pi f_{i j} n\right)
$$

Where

$\mathrm{p}_{\mathrm{i}}=\mathrm{i}$-th binary data

$\mathrm{q}_{\mathrm{i}}(\mathrm{n})=$ multicarrier modulation output of $\mathrm{p}_{\mathrm{i}}$

$r(n)=$ output of multicarrier modulation for several binary data $p_{i}$

$f_{i j}=$ frequency used for modulation, for $i$-th rows and $j$-th column of frequency matrix as 
displayed in figure 4

$\mathrm{N}_{\mathrm{c}}=$ number of same binary data multicarrier modulated (copier)

$\mathrm{N}_{\mathrm{r}}=$ number of rake demultiplexing $(\mathrm{S} / \mathrm{P})$ the binary data $\mathrm{p}_{\mathrm{i}}$

Modulation process consists of pulse shaper, multiplier, oscillator, and adder. Modulation block diagram is shown in figure 5. Frequency allocation for modulation process depends on the number of watermark data. The frequency allocation for 10,20 and 40 bits watermark is shown in Figure 4.

\begin{tabular}{|c|c|c|c|c|c|c|c|c|c|}
\hline & \multicolumn{9}{|c|}{ Copier } \\
\hline & 10 & 510 & 1010 & 1510 & 2010 & 2510 & $\ldots . .$. & 21010 & 21510 \\
\hline & 60 & 560 & 1060 & 1560 & 2060 & 2560 & ......... & 21060 & 21560 \\
\hline & 110 & 610 & 1110 & 1610 & 2110 & 2610 & $\ldots \ldots .$. & 21110 & 21610 \\
\hline & 160 & 660 & 1160 & 1660 & 2160 & 2660 & $\ldots$ & 21160 & 21660 \\
\hline & 210 & 710 & 1210 & 1710 & 2210 & 2710 & ....... & 21210 & 21710 \\
\hline & 260 & 760 & 1260 & 1760 & 2260 & 2760 & …… & 21260 & 21760 \\
\hline & 310 & 810 & 1310 & 1810 & 2310 & 2810 & …... & 21310 & 21810 \\
\hline & 360 & 860 & 1360 & 1860 & 2360 & 2860 & ....... & 21360 & 21860 \\
\hline & 410 & 910 & 1410 & 1910 & 2410 & 2910 & ........ & 21410 & 21910 \\
\hline & 460 & 960 & 1460 & 1960 & 2460 & 2960 & ...... & 21460 & 21960 \\
\hline
\end{tabular}

\section{Copier}

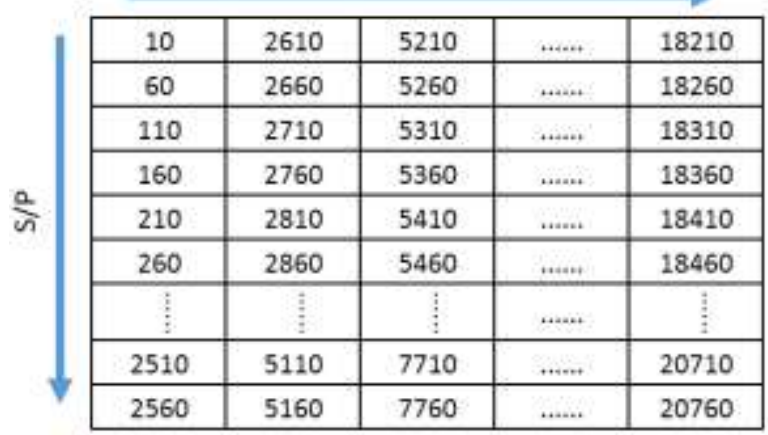

Copier

\begin{tabular}{|r|r|r|r|r|r|r|r|r|r|r|}
\hline 10 & 2010 & 4010 & 6010 & 8010 & 10010 & 12010 & 14010 & 16010 & 18010 & 20010 \\
\hline 60 & 2060 & 4060 & 6060 & 8060 & 10060 & 12060 & 14060 & 16060 & 18060 & 20060 \\
\hline 110 & 2110 & 4110 & 6110 & 8110 & 10110 & 12110 & 14110 & 16110 & 18110 & 20110 \\
\hline 160 & 2160 & 4160 & 6160 & 8160 & 10160 & 12160 & 14160 & 16160 & 18160 & 20160 \\
\hline 210 & 2210 & 4210 & 6210 & 8210 & 10210 & 12210 & 14210 & 16210 & 18210 & 20210 \\
\hline 260 & 2260 & 4260 & 6260 & 8260 & 10260 & 12260 & 14260 & 16260 & 18260 & 20260 \\
\hline 310 & 2310 & 4310 & 6310 & 8310 & 10310 & 12310 & 14310 & 16310 & 18310 & 20310 \\
\hline$\vdots$ & $\vdots$ & $\vdots$ & $\vdots$ & $\vdots$ & $\vdots$ & $\vdots$ & $\vdots$ & $\vdots$ & $\vdots$ & $\vdots$ \\
\hline 1910 & 3910 & 5910 & 7910 & 9910 & 11910 & 13910 & 15910 & 17910 & 19910 & 21910 \\
\hline 1960 & 3960 & 5960 & 7960 & 9960 & 11960 & 13960 & 15960 & 17960 & 19960 & 21960 \\
\hline
\end{tabular}

Figure 4. Frequencies Allocation for 10, 20, and 40 bits respectively [7]

In figure 4 the horizontal number means the frequencies (in $\mathrm{Hz}$ ) which are used to modulate the same bit using copier to replicate the bit. The vertical number means the frequencies which are used to modulate the different bits using serial to parallel to demultiplex the bits. As 
example, from the middle of figure 4 , the 1 st bit of information will be modulated by frequencies $10,2610,5210,7810,10410,13010,15610$, and 18210 . The $2^{\text {nd }}$ bit of the information will be modulated by frequencies 60, 2660, 5260, 7860, 10460, 13060, 15660 and 18260 , until the last bit $\left(20^{\text {th }}\right.$ bit) which will be modulated by frequencies $2560,5160,7760$, $10360,12960,15560,18160$, and 20760. The demodulation process of the watermarked audio needs copier, multiplier, oscillator, adder, low pass filter, integrator, bit sign rounding, and parallel to serial/multiplexer. The block diagram of demodulation process is shown in Figure 6.

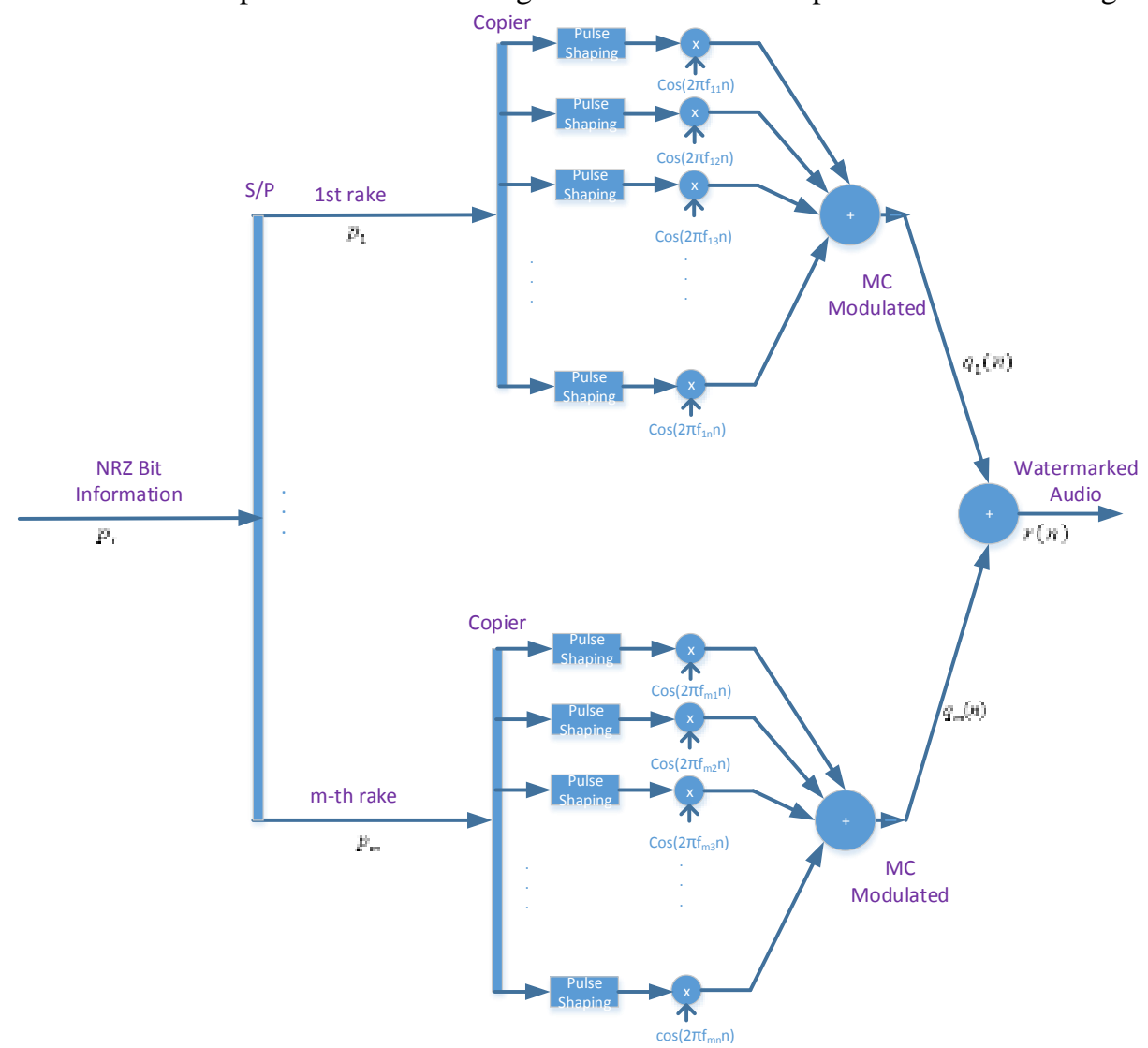

Figure 5. Multicarrier Modulation in Embedding Process of AWS

Demodulation process starts from the cosine multiplication with the same frequency as the modulation process [9] :

$$
\hat{q}_{i}(n)=\hat{r}(n) \sum_{j=1}^{N_{c}} \cos \left(2 \pi f_{i j} n\right)
$$

Then it is filtered by Low Pass Filter, integrated, and detected by threshold detector [9]:

$$
\hat{p}_{i}=\left\{\begin{array}{l}
1, \quad \sum_{i=0}^{N_{s}} \hat{q}_{i}(n) * g_{L P F}(n) \geq 0 \\
-1, \quad \sum_{i=0}^{N_{s}} \hat{q}_{i}(n) * g_{L P F}(n)<0
\end{array}\right.
$$

Where

$\mathrm{N}_{\mathrm{s}}=$ Windowing/Symbol Period of demodulator

$\mathrm{g}_{\mathrm{LPF}}(\mathrm{n})=\mathrm{LPF}$ coefficient 


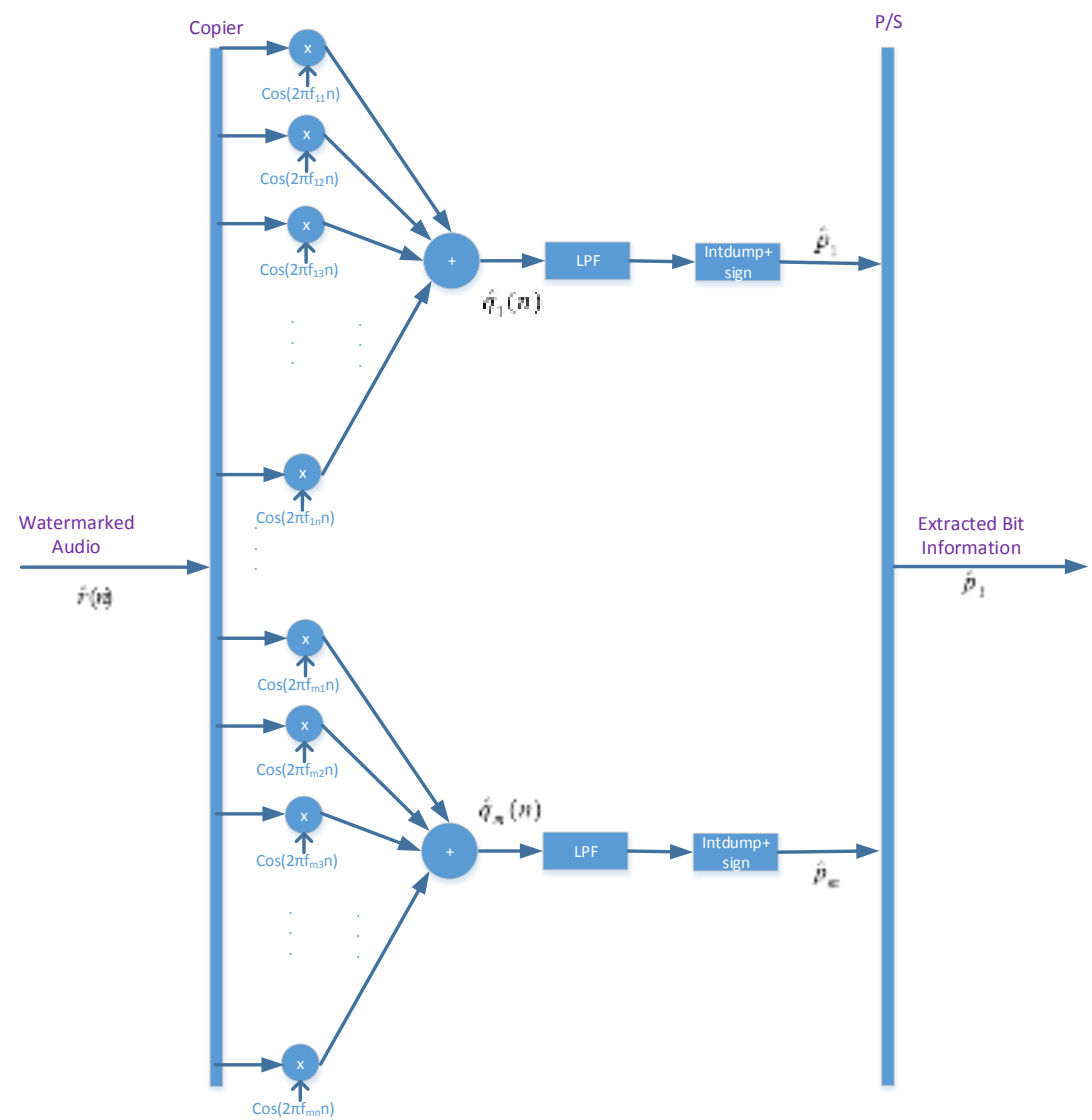

Figure 6. Multicarrier Demodulation

Psychoacoustic filtering is conducted by an Infinite Impulse Response (IIR) filter. The filter coefficients are designed by the required spectrum of psychoacoustic model and approached by pole and zero mapping design as in [10]. The psychoacoustic filter in z-domain is described by this equation :

$$
H(z)=\frac{0.07-0.147 z^{-1}+0.1276 z^{-2}-0.053 z^{-3}+0.0091 z^{-4}}{1-0.15 z^{-1}-0.76 z^{-2}}
$$

From this equation we can get the magnitude response which is similar with the psychoacoustic response characteristic as displayed in Figure 7. This figure describes the magnitude response of psychoacoustic filter model. In this figure the line is audibility threshold which means human can hear only and if only the signal has the amplitude bigger than the magnitude values indicated by the red line. Therefore the hidden data must have the amplitude which is less than the threshold. 


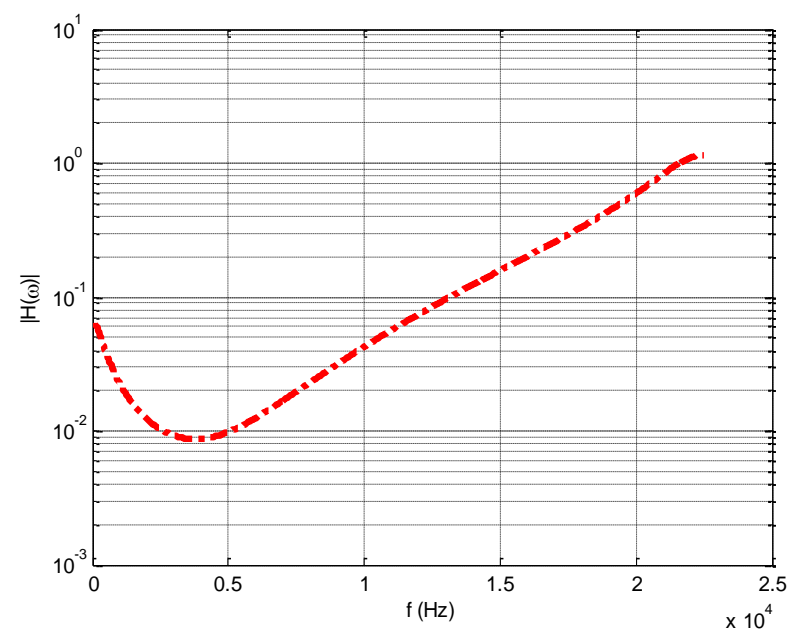

Figure 7. Magnitude response of psychoacoustic filter [10]

\section{Performance Evaluation}

In this section, the proposed method is evaluated in not only its imperceptibility and capacity, but also the robustness of the watermark against several audio signal processing attack. The imperceptibility of the watermark is affected by $A_{1}$ and $A_{2}$ parameter as watermark gain level in the embedding side of the audio watermarking system. The parameter representing imperceptibility are Objective Different Grade (ODG) and Subjective Different Grade (SDG). ODG and SDG will have mark as seen on table 1 as ITU-R BS.1387-1 standard about audio quality. Based on ITU-R standard, ODG is calculated via complex computation on audio signal processing based which named PEAQ (Perceptual Evaluation of Audio Quality) [11]. From table 1 we see that ODG mark has value range from -4 to 0 . In other scale but on linear relation, SDG mark has value range from 1 to 5. SDG is reported by 6 respondents via listening the original and watermarked audio, then they give mark from 1 to 5 as five grade impairment scale seen in table 1 . The average of their mark will be SDG per testing item.

Table 1. ITU-R five grade imperceptibility scale [11]

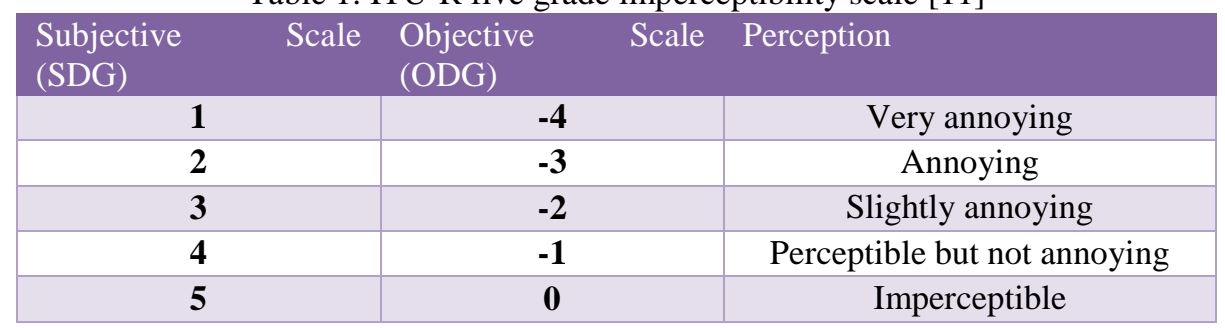

Aside from SDG and ODG, there is robustness parameter which has same importance with imperceptibilty parameter. The parameter is bit error rate or BER. BER is calculated by comparing the original watermark bit and the extracted or detected watermark bit. Usually, BER is calculated after extracting the watermark after the watermarked audio is attacked. But for optimizing the parameters, BER is calculated without watermarked audio attack. Parameter optimized are $A_{1}, A_{2}$, HPF cut off frequency in embedding $\left(f_{c o 1}\right)$ and HPF cut off frequency in extraction side $\left(\mathrm{f}_{\mathrm{co} 2}\right)$. After the optimized parameters are selected, then the audio watermarking system with fixed parameters will be attacked by several attack for watermark robustness measurements. 
A. The effect of gain level on watermarking imperceptibility

In this experiment, we will decide the value of $A_{1}$ and $A_{2}$ for acceptable audio watermarking imperceptibility. Watermark bit number used are $40 \mathrm{bps}$. HPF cut off frequency in embedding side is $10 \mathrm{kHz}$. HPF cut off frequency in extracting side is $4 \mathrm{kHz}$. The duration of the host audio is $3 \mathrm{~s}$. $\mathrm{A}_{1}$ is changed gradually from 0.00008 to 0.03 , and $\mathrm{A}_{2}$ is also set gradually from 0 to 0.01 . Host audio used is "dialogue.wav" with full background music and voice during $3 \mathrm{~s}$.

As shown at table 2, the imperceptibility of watermarked audio or ODG and SDG tend to increase when $A_{1}$ is decrease. $A_{2}$ also will affect the imperceptibility, when $A_{2}$ is increased, then ODG and SDG tend to decrease. But for ODG, the value is slightly fluctuative. Thus, we can choose SDG for more valid parameter for decision. Highest $A_{1}$ and $A_{2}$ for accepted imperceptibility is obtained when $\mathrm{A}_{1}=0.009$ and $\mathrm{A}_{2}=0$ ( $\mathrm{SDG}=4.5$ ), or $\mathrm{A}_{1}=0.01$ and $\mathrm{A}_{2}=0.002$ (SDG=3.67). We select highest combination $A_{1}$ and $A_{2}$ for next testing in order to make it robust to the watermarked audio attack, but at the same time its imperceptibility is still acceptable. For next experiment parameters used for the attack testing are $A_{1}=0.01$ and $\mathrm{A}_{2}=0.001$. Relatively the SDG will still be acceptable for that value of $\mathrm{A}_{1}$ and $\mathrm{A}_{2}$. From table 2 it can be seen that there is a minimum value of $A_{1}$ for keeping the watermark extraction without error. $\mathrm{A}_{1}=0.0004$ is minimum limit for keeping $\mathrm{BER}=0$ with no attack in this experiment.

Table 2. Imperceptibility and initialization of robustness testing at host audio "dialogue.wav"

\begin{tabular}{|c|c|c|c|c|c|}
\hline $\mathbf{A}_{\mathbf{1}}$ & $\mathbf{A}_{\mathbf{2}}$ & ODG & SNR & SDG & BER \\
\hline $\mathbf{0 . 0 0 0 0 8}$ & 0 & -0.26 & 49.91 & 5 & 0.15 \\
\hline $\mathbf{0 . 0 0 0 9}$ & 0 & -0.3 & 48.89 & 5 & 0.16 \\
\hline $\mathbf{0 . 0 0 0 1}$ & 0 & -0.28 & 47.98 & 5 & 0.12 \\
\hline $\mathbf{0 . 0 0 0 1 1}$ & 0 & -0.3 & 47.15 & 5 & 0.12 \\
\hline $\mathbf{0 . 0 0 0 2}$ & 0 & -0.44 & 41.95 & 5 & 0.08 \\
\hline $\mathbf{0 . 0 0 0 3}$ & 0 & -0.6 & 38.44 & 5 & 0.08 \\
\hline $\mathbf{0 . 0 0 0 4}$ & 0 & -0.65 & 35.94 & 5 & 0 \\
\hline $\mathbf{:}$ & $:$ & $:$ & & $:$ & $:$ \\
\hline $\mathbf{:}$ & $:$ & $:$ & & $:$ & $:$ \\
\hline $\mathbf{0 . 0 0 8}$ & 0 & -2.24 & 10.34 & 4.50 & 0 \\
\hline $\mathbf{0 . 0 0 9}$ & 0 & -2.3 & 9.42 & 4.50 & 0 \\
\hline $\mathbf{0 . 0 1}$ & 0 & -2.2 & 8.62 & 3.50 & 0 \\
\hline $\mathbf{0 . 0 2}$ & 0 & -2.2 & 4.1 & 2.83 & 0 \\
\hline $\mathbf{0 . 0 3}$ & 0 & -2.22 & 2.3 & 2.50 & 0 \\
\hline $\mathbf{0 . 0 1}$ & 0.01 & -2.79 & 4.1 & 2.83 & 0 \\
\hline $\mathbf{0 . 0 1}$ & 0.008 & -2.2 & 4.68 & 2.83 & 0 \\
\hline $\mathbf{0 . 0 1}$ & 0.006 & -2.52 & 5.38 & 3.33 & 0 \\
\hline $\mathbf{0 . 0 1}$ & 0.004 & -2.2 & 6.24 & 3.33 & 0 \\
\hline $\mathbf{0 . 0 1}$ & 0.002 & -2.26 & 7.3 & 3.67 & 0 \\
\hline
\end{tabular}

B. The effect of watermark payload on watermarking performance

In this experiment, audio file used for embedding and extraction is "dialogue.wav". Watermark data is generated on randomly uniform distribution. Watermark bit number is set to be $10 \mathrm{bps}$ and $40 \mathrm{bps}$ for performance comparison. $\mathrm{A}_{2}$ is set to be 0 . Only $\mathrm{A}_{1}$ is changed from 0.00006 to 0.0004 . The result is displayed on table 3 . It can be seen that limit of perfect BER 
and error BER will increase since the watermark payload increase. When bit number is $10 \mathrm{bps}$, BER will be no longer zero at $A_{1}=0.00008$, but when bit number is $40 \mathrm{bps}$, BER will be no longer zero at $A_{1}=0.0004$. This means that the robustness of watermark will decrease since the watermark payload increases. The payload is inversely proportional with the robustness.

Table 3. Bit number effect on imperceptibility and robustness at "dialogue.wav"

\begin{tabular}{|c|c|c|c|c|}
\hline Bit Number & A1 & ODG & SDG & BER \\
\hline \multirow{9}{*}{10 bps } & 0.00006 & -0.21 & 5 & 0.1 \\
\hline & 0.00007 & -0.25 & 5 & 0.07 \\
\hline & 0.00008 & -0.26 & 5 & 0.03 \\
\hline & 0.00009 & -0.27 & 5 & 0 \\
\hline & 0.0001 & -0.29 & 5 & 0 \\
\hline & 0.00011 & -0.31 & 5 & 0 \\
\hline & 0.0002 & -0.44 & 5 & 0 \\
\hline & 0.0003 & -0.59 & 5 & 0 \\
\hline & 0.0004 & -0.89 & 5 & 0 \\
\hline \multirow{9}{*}{40 bps } & 0.00006 & -0.2 & 5 & 0.19 \\
\hline & 0.00007 & -0.26 & 5 & 0.09 \\
\hline & 0.00008 & -0.26 & 5 & 0.15 \\
\hline & 0.00009 & -0.3 & 5 & 0.16 \\
\hline & 0.0001 & -0.28 & 5 & 0.12 \\
\hline & 0.00011 & -0.3 & 5 & 0.12 \\
\hline & 0.0002 & -0.44 & 5 & 0.08 \\
\hline & 0.0003 & -0.6 & 5 & 0.08 \\
\hline & 0.0004 & -0.65 & 5 & 0 \\
\hline
\end{tabular}

\section{Testbed Result Performance}

The testbed procedure by several attack types has several points of testing which generally consists of lowpass filtering, bandpass filtering, noise addition, resampling, time scale modification, linear speed change, pitch shifting, multi band equalizing, echo addition, and MP3 compression. The attack type and their description are displayed in table 4. Cut off frequency for LPF attack are set to $6,9,12,16 \mathrm{kHz}$. High cut off frequency for BPF attack are also set to $6,9,12,16 \mathrm{kHz}$. Noise additive will add the noise to the watermarked audio in cascade additional between white noise and pink noise. The level noise is set to $20 \mathrm{~dB}$ below average watermarked audio power. Resampling attack is set to $22.05 \mathrm{kHz}, 16 \mathrm{kHz}$, and 11.025 $\mathrm{kHz}$. Linear speed change attack are set to $-15 \%,-10 \%,-5 \%, 5 \%, 10 \%$, and $15 \%$. Multi band equalizer attack will have 10 band graphic and consists of band frequency $(\mathrm{Hz})$ : [ [31 62125 $2505001 \mathrm{kHz} 2 \mathrm{kHz} 4 \mathrm{kHz} 8 \mathrm{kHz} 16 \mathrm{kHz}]$ and gain (dB) [-6 +6 -6 +6 -6 +6 -6 +6 -6 +6 ]. Echo addition attack will have maximum delay $100 \mathrm{~ms}$ and feedback coefficient around 0.3. And last attack is MP3 compression which has rate : $32 \mathrm{kbps}, 64 \mathrm{kbps}, 128 \mathrm{kbps}, 192 \mathrm{kbps}$, and $256 \mathrm{kbps}$. The host audio used for embedding, attacking, and extraction consist of 5 audio files, that is "dialogue.wav", "fleetwd.wav", "Moonriver_Mancini.wav", "mouth_harmonica.wav", and "Sax_Piano.wav". Each host audio file has duration $3 \mathrm{~s}$. Watermark data is generated on randomly uniform distribution at 10 and 40 bps payload. 
Table 4. Testbed list as watermarked audio attack

\begin{tabular}{|c|c|c|}
\hline Item & Type of attack & Attack Description \\
\hline 1 & Low pass filter & $\begin{array}{l}\text { cut off frequency }=9,12,16 \mathrm{kHz} \text {, second order butterworth } \\
\text { filter }\end{array}$ \\
\hline 2 & Band pass filter & $\begin{array}{l}\text { cut off frequency }=100 \mathrm{~Hz}-9 \mathrm{kHz}, 100 \mathrm{~Hz}-12 \mathrm{kHz}, 100 \mathrm{~Hz}- \\
16 \mathrm{kHz}, 2 \text { nd order Butterworth filter }\end{array}$ \\
\hline 3 & Noise addition & $\begin{array}{l}\text { Adding white and pink noise with constant level of } 20 \mathrm{~dB} \\
\text { lower than total averaged music power }\end{array}$ \\
\hline 4 & $\begin{array}{l}\text { Changing } \\
\text { sample rate }\end{array}$ & $\begin{array}{l}44.1 \mathrm{kHz}->22.05 \mathrm{kHz}, 44.1 \mathrm{kHz}->16 \mathrm{kHz}, 44.1 \mathrm{kHz}-> \\
11.025 \mathrm{kHz}\end{array}$ \\
\hline 5 & $\begin{array}{l}\text { Linear speed } \\
\text { change }\end{array}$ & $-15 \%,-10 \%,-5 \%, 5 \%, 10 \%, 15 \%$ \\
\hline 6 & $\begin{array}{l}\text { Multi-band } \\
\text { equalization }\end{array}$ & $\begin{array}{l}\text { 10-band graphic equalizer with the characteristics listed below: } \\
\text { Freq.[Hz]: } 31621252505001 \mathrm{kHz} 2 \mathrm{kHz} 4 \mathrm{kHz} 8 \mathrm{kHz} 16 \mathrm{kHz} \\
\text { Gain[dB]: }-6+6-6+6-6+6-6+6-6+6\end{array}$ \\
\hline 7 & Echo addition & $\begin{array}{l}\text { Maximum } \quad \text { delay: } \\
\text { Feedback coefficient: around } 0.3\end{array}$ \\
\hline 8 & MP3 Compression & $32,64,128,192,256 \mathrm{kbps}$ \\
\hline
\end{tabular}

The used parameters for testbed : $A_{1}=0.01, A_{2}=0.001$, HPF cut off frequency in embedding side is $10 \mathrm{kHz}$ in order to keep good imperceptibility, HPF cut off frequency in extracting side is $4 \mathrm{kHz}$ to keep the good robustness, especially when the watermarked audio faces low pass filtering attack with $6 \mathrm{kHz}$ cut off frequency. Watermark payload used are $10 \mathrm{bps}$ and $40 \mathrm{bps}$. The overall results displayed in table 5 are average of bit error rate from each file in every attack type and every parameter of attack.

Table 5. Testbed result

\begin{tabular}{|c|c|c|c|c|}
\hline Item & Type of attack & $\begin{array}{l}\text { Payload } \\
\text { (bps) }\end{array}$ & Parameters & Average BER \\
\hline \multirow{2}{*}{1} & \multirow{2}{*}{ Low pass filter } & 10 & $\mathrm{fco}=6,9,12,16 \mathrm{kHz}$ & $0.34,0.27,0.09,0.007$ \\
\hline & & 40 & $\mathrm{fco}=6,9,12,16 \mathrm{kHz}$ & $0.47,0.37,0.17,0.03$ \\
\hline \multirow{2}{*}{2} & \multirow{2}{*}{ Band pass filter } & 10 & $f c o=100 \mathrm{~Hz}-6 / 9 / 12 / 16 \mathrm{kHz}$ & $0.34,0.27,0.09,0.007$ \\
\hline & & 40 & $\mathrm{fco}=100 \mathrm{~Hz}-6 / 9 / 12 / 16 \mathrm{kHz}$ & $0.48,0.37,0.17,0.03$ \\
\hline 3 & Noise addition & 10,40 & $\begin{array}{l}\text { White and pink noise with } 20 \mathrm{~dB} \text { lower than averaged } \\
\text { audio power }\end{array}$ & 0,0 \\
\hline \multirow{2}{*}{4} & \multirow{2}{*}{$\begin{array}{l}\text { Changing the } \\
\text { sample rate }\end{array}$} & 10 & $22.05,16,11.025 \mathrm{kHz}$ & $0.0067,0.0067,0$ \\
\hline & & 40 & $22.05,16,11.025 \mathrm{kHz}$ & $0.005,0,0.002$ \\
\hline \multirow{2}{*}{5} & \multirow{2}{*}{$\begin{array}{l}\text { Linear speed } \\
\text { change }\end{array}$} & 10 & $-15,-10,-5,5,10,15 \%$ & $0,0,0,0,0,0$ \\
\hline & & 40 & $-15,-10,-5,5,10,15 \%$ & $0,0,0,0,0,0$ \\
\hline 6 & $\begin{array}{l}\text { Multi-band } \\
\text { equalization }\end{array}$ & 10,40 & $\begin{array}{l}\text { 10-band graphic equalizer with the characteristics } \\
\text { listed below: } \\
\text { Freq.[Hz]: } 31621252505001 \mathrm{kHz} 2 \mathrm{kHz} 4 \mathrm{kHz} 8 \mathrm{kHz} \\
16 \mathrm{kHz} \\
\text { Gain[dB]: }-6+6-6+6-6+6-6+6-6+6\end{array}$ & 0,0 \\
\hline 7 & Echo addition & 10,40 & $\begin{array}{l}\text { Maximum delay: } 100 \mathrm{~ms} \\
\text { Feedback coefficient: around } 0.3\end{array}$ & 0,0 \\
\hline \multirow{2}{*}{8} & \multirow{2}{*}{$\begin{array}{l}\text { MP3 } \\
\text { Compression }\end{array}$} & 10 & $32,64,128,192,256 \mathrm{kbps}$ & $0.47,0.09,0,0,0$ \\
\hline & & 40 & $32,64,128,192,256 \mathrm{kbps}$ & $0.48,0.2,0,0,0$ \\
\hline
\end{tabular}


From table 5, it can be seen that overall audio watermarking in multicarrier-based with the optimized parameter as explain in subsection IV.A obtains good result. The perfect robustness results with BER 0 are obtained in noise addition attack, linear speed change attack, multi band equalization attack, and echo addition attack. Audio watermarking robustness in LPF and BPF attack is similar. The robustness tends to be better when the cut off frequency of LPF and BPF increases. The watermark robustness from last attack, MP3 compression, has acceptable result, except at compression rate $32 \mathrm{kbps}$. The robustness from MP3 compression attack is perfect when MP3 compression rate is more closely with $128 \mathrm{kbps}$. Especially for $10 \mathrm{bps}$ payload, this technique of audio watermarking has acceptable robustness with MP3 compression attack for MP3 compression rate more closely with 64 kbps.

Table 6. Robustness comparison with different method

\begin{tabular}{|c|c|c|c|c|c|}
\hline \multirow{2}{*}{ Scheme } & \multirow{2}{*}{ LPF } & \multicolumn{2}{|c|}{ Resampling } & \multicolumn{2}{c|}{ MP3 Compression } \\
\cline { 3 - 6 } & & $\mathbf{1 1 . 0 2 5} \mathbf{~ k H z}$ & $\mathbf{2 2 . 0 5} \mathbf{~ k H z}$ & $\mathbf{6 4} \mathbf{k b p s}$ & $\mathbf{1 2 8} \mathbf{k b p s}$ \\
\hline$[12]$ & $0 \%(18 \mathrm{kHz})$ & NA & NA & $9 \%$ & $10 \%$ \\
\hline$[13]$ & $\begin{array}{c}1.43 \% \\
(6 \mathrm{kHz})\end{array}$ & NA & 0 & $1.43 \%$ & NA \\
\hline$[14]$ & NA & NA & 0 & NA & $3.30 \%$ \\
\hline$[15]$ & NA & NA & 0 & NA & $2.93 \%$ \\
\hline $\begin{array}{c}\text { Proposed } \\
(40 \text { bps payload })\end{array}$ & $3 \%(16 \mathrm{kHz})$ & $0.20 \%$ & $0.50 \%$ & $20 \%$ & $0 \%$ \\
\hline $\begin{array}{c}\text { Proposed } \\
(10 \text { bps payload })\end{array}$ & $\begin{array}{c}0.7 \%(16 \\
\mathrm{kHz})\end{array}$ & $0.67 \%$ & $0 \%$ & $9 \%$ & $0 \%$ \\
\hline
\end{tabular}

Table 6 displays robustness comparison with last different method of frequency domain based audio watermarking, but not all method described the robustness of the same attack, thus several attacks obtained NA (not available) robustness. Mehdi in [12] used Fibonacci as sequence for embedding watermark into host audio in frequency domain by FFT. His method obtained perfect watermark with zero BER when the watermarked audio was attacked by LPF, but the cut off frequency was set to $18 \mathrm{kHz}$. Mehdi obtained 10\% BER when the audio watermarked was attacked by 128 kbps MP3 compression. Yiqing [13] used FFT as transform domain and psychoacoustic model with gammatone filter for embedding the watermark. She also used artificial intelligence after extracting the watermark to get binary based watermark data, thus she obtained the robustness better than ours in LPF attack, and MP3 compression. Pranab [14] used DWT-DCT-SVD method to embed and extract the watermark in audio watermarking. And he also published another method in [15] using FFT-SVD-CPT method. Anyway, our method has reached perfect robustness when the watermarked audio files are attacked by $128 \mathrm{kbps}$ MP3 compression, while the other method couldn't reach that performance.

\section{Conclusion}

The testing result of multicarrier modulation shows that information bit hidden in the host audio could reach up to $40 \mathrm{bps}$ and the imperceptibility level is still acceptable, due to MOS result is more than 4 . The testbed procedure result gives the perfect robustness in noise addition attack, linear speed change attack, multi band equalization attack, and echo addition attack. And the robustness of audio watermarking is still acceptable with MP3 compression attack and filtering for cut off frequency up to $16 \mathrm{kHz}$, due to BER is lower than $10 \%$. Comparing with other current method, this proposed method has perfect robustness or zero BER when the attack type used 128 kbps MP3 compression. 


\section{Acknowledgements}

This research was partially supported by ITB WCU grant 2016 and RISET ITB 2016

\section{Bibliography}

[1]. R. Van Nee and R. Prasad, OFDM for Wireless Multimedia Communications. London: Artech House, 2000

[2]. N. Marchetti, M. I. Rahman, and S. Kumar, New Directions in Wireless Communications Research. 2009

[3]. S. Maiti, A. Roy, T. S. Das, and S. K. Sarkar, "OFDM Based High Capacity Information Hiding in Grey Scale Image," International Journal of Soft Computing and Engineering (IJSCE), vol. 1, no. 6, pp. 192-195, 2012

[4]. R. Amirtharajan, K. Thenmozhi, and R. J. B. Balaguru, "Multi Carrier Steg against Omni Attacks," International Journal of Computer Applications, vol. 5, no. 11, pp. 35-40, 2010

[5]. A. V Shishkin, "OFDM-based Audio Watermarking for Electronic Radiotelephone Identification,” Design \& Test Symposium (EWDTS), 2010 East-West, pp. 190-194, 2010

[6]. J. J. Garcia-Hernandez, R. Parra-Michel, C. Feregrino-Uribe, and R. Cumplido, "High payload data-hiding in audio signals based on a modified OFDM approach," Expert Systems with Applications, vol. 40, no. 8, pp. 3055-3064, 2013

[7]. G. Budiman, A. B. Suksmono, and D. H. Shin, "A Multicarrier Modulation Audio Watermarking System," in International Conference on Electrical Engineering and Informatics 2015, 2015, no. 2, pp. 164-171

[8]. K. Fazel and S. Kaiser, Multi-Carrier and Spread Spectrum Systems. 2003

[9]. R. E. Z. (University of Colorado) and W. H. T. (Virginia P. I. and S. University), Principles of Communications: System, Modulation and Noise, 6th ed. John Wiley and Sons, 2010

[10]. D. J. M. Robinson, "the Human Auditory System," 107th convention of the Audio Engineering Society, pp. 1-13, 1999

[11]. R. ITU-R, RECOMMENDATION ITU-R BS.1387-1 Method for objective measurements of perceived audio quality, vol. BS.1387-1. 1998

[12]. M. Fallahpour and D. Megías, "Audio Watermarking Based on Fibonacci Numbers," IEEE/ACM Transactions on Audio, Speech, and Language Processing, vol. 23, no. 8, pp. $1273-1282,2015$

[13]. Y. Lin and W. H. Abdulla, Audio Watermark, A Comprehensive Foundation Using Matlab. Auckland: Springer, 2015

[14]. P. K. Dhar and T. Shimamura, Advances In Audio Watermarking Based on SVD. New York, London: Springer, 2015

[15]. P. K. Dhar and T. Shimamura, "Audio watermarking in transform domain based on singular value decomposition and Cartesian-polar transformation," International Journal of Speech Technology, vol. 17, no. 2, pp. 133-144, 2014

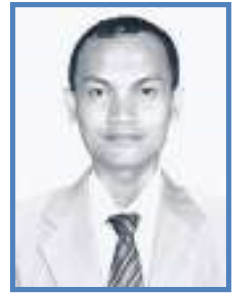

Gelar Budiman received B.S. and M.S. degree in electrical engineering from Sekolah Tinggi Teknologi Telkom (STTT), Bandung, Indonesia. He has been working as a lecturer in Telkom University since 2008. He has been currently taking doctoral degree in School of Electrical Engineering and Informatics, Bandung Technology Institute (ITB), since 2015. His research interests are in the areas of wireless communication, signal processing, and watermarking. 


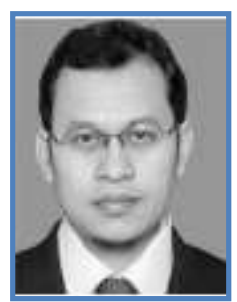

Andriyan Bayu Suksmono (M'02-SM'08) received the B.S. degree in physics and the M.S. degree in electrical engineering from the Bandung Institute of Technology (ITB), Indonesia, and the Ph.D. degree in engineering from the University of Tokyo, Japan, in 1990, 1996 and 2002, respectively. He joined ITB as an Instructor (1996-2005), Associate Professor (2005-2009), and Professor (2009-present) at the School of Electrical Engineering and Informatics, ITB. His main research interests are signal processing and imaging.

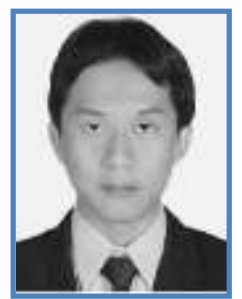

Donny Danudirdjo received the B.S. and M.S. degrees in electrical engineering from the Bandung Institute of Technology (ITB), Bandung, Indonesia, in 2005 and 2007 and the Ph.D. degree from The University of Tokyo, Tokyo, Japan, in 2013.

Since 2014, he has been with the School of Electrical Engineering and Informatics, ITB. His current research interests include interferometric synthetic aperture radar image analysis and fractal-related topics in signal and image processing.

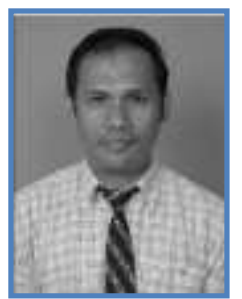

Koredianto Usman was born in Sumatera Selatan Indonesia October 2, 1975. His obtained his bachelor from Electrical Engineering Bandung Institute of Technology, Indonesia, 1999, and master degree from Munich Institute of Technology, Germany in 2001. He is now a researcher at School of Electrical Engineering and Informatics, Bandung Technology Institute (ITB).

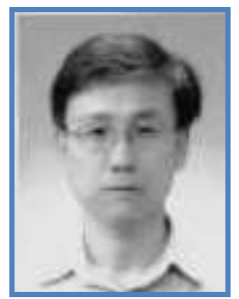

Dong Hwan Shin received the MS degree and Ph.D. in electronics engineering from University of Seoul, Korea. From 1992 to 1994, he was a member of the LG Electronics Inc. He worked as a senior researcher in the Korea Sports Science Institute from 1996 to 2000. He has been currently a chief manager of MarkAny Inc. from 2000. Her research interests are in the areas of copyright protection, fingerprinting, watermarking and machine learning. 